\title{
MINIMAL GENERATING SETS FOR FREE MODULES
}

\section{M. BRUNING AND W. G. LEAVITT}

Abstract. Let $R$ be a ring admitting a free module with generating set shorter than the length of a basis. If $n$ is the shortest basis among all such modules and $m$ the length of its shortest generating set then $n=m+1$ and every free module with basis of length $\geqq m+1$ has a generating set of length $m$. If $R$ has module type $(h, k)$ then $m=h$, that is an $R$-module with basis of length $u<h$ not only has all bases of length $u$ but also has no generating set of length $<u$. The integer $m$ together with the module type define a new ring invariant which satisfies many of the properties of the module type.

1. Introduction. In the following, we will consider only rings with unit. A finitely based module over a ring is said to have rank $t$, if it has a basis of length $t$. A module may not necessarily have unique rank. Modules with unique rank are said to have dimension and a ring admitting only such modules is termed dimensional. The second author [2, p. 114, Theorem 1] has shown that a given ring admits only certain characteristic finitely based modules; that is, for any nondimensional ring $R$ there exist unique positive integers, $h, k$, such that (i) any $R$-module $M$ with a basis of length $<h$ has dimension; (ii) for any $R$-module $M$ with a basis of length $\geqq h$ there exists an integer $p$, where $h \leqq p<h+k$ such that $M$ has a basis of length $r$ if and only if $r=p+u k$ for some integer $u \geqq 0$. Moreover, such an $R-$ module exists for arbitrary $p$. Thus an $R$-module $M$ with rank $<h$ is isomorphic only to a module with the same rank, while if its rank is $p \geqq h$ then $M \cong M^{\prime}$ if and only if $M^{\prime}$ has $\operatorname{rank} r \geqq h$ with $r \equiv p(\bmod k)$. Such a ring is said to have module type $(h, k)$. Dimensional rings are designated module type $d$.

Certain rings admit finitely based modules of rank $t$ which can be generated by fewer than $t$ elements. Clearly all nondimensional rings have this property. P. M. Cohn $[1$, p. 221] has given an example of a dimensional ring which has this property, but shows that no module of rank $t$ of a Noetherian ring, Artinian ring, or commutative ring can be generated by fewer than $t$ elements. He also proposes a classification of rings admitting a free module with a set of generators shorter than the length of a basis by the two integers $m$ and $n$, where

Received by the editors April 8, 1970.

AMS 1969 subject classifications. Primary 1640, 1650.

Key words and phrases. Generators of free modules, rank of a module, module type. 
$n$ is the least rank of a free module which can be generated by fewer than $n$ elements and $m$ is the least number of elements required to generate it.

In this paper, we will investigate (for such a ring) the possibilities for $m$ and $n$. Our main tool will be a certain epimorphism theorem of P. M. Cohn $[1$, p. 216]. We will show first that $n$ must be equal to $m+1$ and that if a module has a rank $t \geqq m+1$, the module has a minimal generating set of length $m$. In the special case where the ring is nondimensional, that is, has module type $(h, k)$, we find that no module of rank less than $h$ can be generated by fewer elements than the length of a basis, and thus it follows that $m=h$.

In the final section, a classification of rings by $m$, the least number of elements required to generate a module of greater rank, and the module type of a ring is developed. This classification has a natural partial order imposed by the partial ordering of the module types, and is shown to satisfy many of the theorems valid for the module type.

2. Determination of the length of minimal generating sets. Clearly any module having a basis of length $t$ is isomorphic to any other module having a basis of length $t$. We designate such a module by $F_{t}$. We will use the result $[1$, p. 216]:

Theorem A (CoHN). $F_{t}$ has a generating set of length $m$ if and only if $F_{m} \cong F_{t} \oplus K$ for some $R$-module $K$.

Let $\delta$ be the class of all $R$-modules, $M$, such that for some $k, M$ has a generating set of length $k$ and a basis of length greater than $k$. Let $m$ and $n$ be positive integers with the property:

(i) $n$ is the length of the shortest basis of any $M \in S$ which can be generated by fewer than $n$ elements, and $m$ is the least number of elements required to generate $F_{n}$.

Theorem 1. Let $R$ be a ring with nonempty $\delta$. Suppose $m$ and $n$ have property (i), then $n=m+1$.

Proof. $n>m$, therefore $n \geqq m+1$. Suppose $n>m+1$. Since $F_{m+1}$ is isomorphic to the module generated by the first $m+1$ elements of a basis of $F_{n}$ and $F_{n-(m+1)}$ is isomorphic to the module generated by the remainder of the basis of $F_{n}$, we have $F_{n} \cong F_{m+1} \oplus F_{n-(m+1)}$. But by Theorem $\mathrm{A}$ this implies that $F_{m+1}$ has a generating set of length $m$ because $F_{n}$ does. Therefore $F_{m+1}$ belongs to $s$. Since $n$ is the length of the smallest basis of any module belonging to $\delta, n=m+1$. 
THEOREM 2. Let $R$ have nonempty $s$ and $n$ and $m$ have property (i), then if $t \geqq n, F_{t}$ has a minimal generating set of length $m$.

Proof. By Theorem 1 and the definition of $m$ and $n$, we have that $F_{n}=F_{m+1}$ has a minimal generating set of length $m$ and hence there exists by Theorem A an epimorphism from $F_{m}$ onto $F_{m+1}$. Assume for induction that $F_{m+k}$ has a minimal generating set of length $m$. Then $F_{m+k+1} \cong F_{m+k} \oplus F_{1}$ has a generating set of length $m+1$. Thus $F_{m+k+1}$ is the epimorphic image of $F_{m}$ and hence has a minimal generating set of length $m$. The result thus follows by induction.

We now consider a ring $R$ with module type $(h, k)$. We will show that $m=h$, that is, no $R$-module with a basis of length $u \leqq h$ can be generated by fewer than $u$ elements. On the other hand, those modules with bases of length $u>h$ have minimal generating sets of length $h$.

Leмma 1. Let $R$ be of module type $(h, k)$ and let $r$ be the minimum length of a generating set for $F_{h}$. Whenever $F_{t}$ has a minimal generating set of length $s<t$ then $r=s<t$.

Proof. First, suppose $t<r \leqq h$. We have $F_{r} \cong F_{t} \oplus F_{r-t}$ and $F_{r} \cong F_{h}$ $\oplus A$ for some $A$. Also $F_{s} \cong F_{t} \oplus B$ for some $B$ and therefore $F_{s} \oplus F_{r-t}$ $\cong F_{h} \oplus A \oplus B$. But, $F_{s} \oplus F_{r-t}$ has a basis of length $s+(r-t)$, hence $F_{h}$ has a generating set of length $s+(r-t)<r$. This contradicts the minimality of $r$, and so we conclude that $r \leqq t$.

If $r=t$, then $F_{s} \cong F_{t} \oplus B \cong F_{r} \oplus B \cong F_{h} \oplus A \oplus B$ and again $r \leqq s$ by the minimality of $r$. Actually this case is impossible, since $s<t$.

We therefore have $r<t$. By a similar argument to that above, we have $F_{s} \cong F_{t} \oplus B \cong F_{r} \oplus F_{t-r} \oplus B \cong F_{h} \oplus A \oplus F_{t-r} \oplus B$. Therefore $F_{h}$ has a generating set of length $s$. By the minimality of $r$ this implies $r \leqq s$.

Now suppose $h \geqq t$, then $F_{r} \cong F_{h} \oplus A \cong F_{t} \oplus F_{h-t} \oplus A$, which implies that $F_{t}$ has a generating set of length $r$. Therefore $s \leqq r$. On the other hand, if $h<t$, there exists an integer $u$ such that $t<h+u k . F_{h+u k} \cong F_{t}$ $\oplus F_{h+u k-t}$ and $F_{h+u k} \cong F_{h}$, since $R$ has module type $(h, k)$. Thus $F_{t}$ has again a generating set of length $r$. In either case it follows that $r=s$.

Note, that by the definition of $m$ in (i), this lemma shows that $m=r$. We will now show that actually $m=h$.

THEOREM 3. Let $R$ be of module type $(h, k), m$ as in (i), and $r$ the minimum length of a generating set for $F_{h}$, then $m=r=h$.

Proof. (Clearly $r \leqq h$.) $F_{h}$ has a generating set of length $r$, therefore $F_{r} \cong F_{h} \oplus A$ for some $A$. Now, since $R$ has module type $(h, k)$, we have $F_{h+k} \cong F_{h}$. Therefore $F_{r+k} \cong F_{h} \oplus A \oplus F_{k} \cong F_{h+k} \oplus A \cong F_{h} \oplus A \cong F_{r}$. 
But by the definition of module type this implies that $r \geqq h$, and hence we have $r=h$. Thus by the lemma $m=r=h$.

3. The module class of a ring. It is thus clear by Theorems 2 and 3 that for a ring $R$ with nonempty $S$, there is a unique integer $m$ (equal to $h$, when $R$ has type $(h, k)$ ) such that no $R$-module with a basis of length $u \leqq m$ can be generated by fewer than $u$ elements, whereas a module with a basis of length $u>m$ has a minimal generating set of length $m$. We will call $(m, t)=C(R)$ the module class of a ring $R$, where $m$ is this unique integer and $t$ is the module type of $R$. We also set $C(R)=D$ when $\delta$ is empty, that is, when no $F_{r}$ is generated by fewer than $r$ elements, and $C(R)=0$ when $R$ is the ring with 0 as its only element.

The following partial order was established for module types in [2]. (i) $0<(h, k)<d$ for all $(h, k)$ and (ii) $(h, k) \leqq\left(h^{\prime}, k^{\prime}\right)$ if and only if $h \leqq h^{\prime}$ and $k \mid k^{\prime}$. Using this partial ordering, we may partially order module classes by $0<(m, t)<D$ for all module classes $(m, t)$, and $(m, t) \leqq\left(m^{\prime}, t^{\prime}\right)$ if and only if both $m \leqq m^{\prime}$ and $t \leqq t^{\prime}$. Note, that when $t^{\prime}<d$, the condition $t \leqq t^{\prime}$ automatically implies $m \leqq m^{\prime}$.

In [2], examples are given of rings of arbitrary module type and hence of all classes $(m, t)$ where $t<d$. The ring $U_{m, n}$ constructed by Cohn $[1$, p. 221] is clearly of class $(m, d)$. Since, for example, fields have class $D$, we thus have examples of rings of all module classes.

Now it is well known that the existence of an $R$-module with a generating set of length $m$ and a basis of length $n$ is equivalent to the existence of $m$ by $n$ and $n$ by $m$ matrices $C$ and $D$ over $R$ such that $D C=I_{n}$, where $I_{n}$ is the identity matrix. From this observation, the following propositions easily follow and will be stated without proof.

Proposition 1 (COHN). If $\phi: R \rightarrow R^{\prime}$ is a unit-preserving homomorphism then $C\left(R^{\prime}\right) \leqq C(R)$ where $C\left(R^{\prime}\right)$ and $C(R)$ are the respective module classes of $R^{\prime}$ and $R$. Thus $C\left(R^{\prime}\right)=D$ implies $C(R)=D$.

Proposition 2. If $R$ is a subring of a ring $R^{\prime}$, with the same unit, then $C\left(R^{\prime}\right) \leqq C(R)$.

Proposition 3. If $R^{\prime}$ is the ring of polynomials over $R$ in a set $\left\{x_{i}\right\}_{i \in I}$ of symbols, then $C\left(R^{\prime}\right)=C(R)$.

PRoposition 4. If $I$ is a right (left) ideal of $R$ and $I$ is itself a ring with unit, then $C(I) \leqq C(R)$.

In the following theorem, it is shown that we can have $C\left(R_{1}\right)$ not equal to $C(R)$ where $R \cong R_{1} \oplus R_{2}$. Thus the condition in Proposition 1 that $\phi$ be unit-preserving cannot be removed. 
THEOREM 4. If $R \cong R_{1} \oplus R_{2}$ is a ring direct sum with $C\left(R_{i}\right)=\left(m_{i}, t_{i}\right)$ $(i=1,2)$ then $C(R)=\left(\max \left(m_{1}, m_{2}\right), t_{1} \cup t_{2}\right)$. If either $C\left(R_{i}\right)=D$ then $C(R)=D$.

Proof. The natural homomorphisms of $R$ onto $R_{1}$ and $R_{2}$ require that $C\left(R_{1}\right) \leqq C(R)$ and $C\left(R_{2}\right) \leqq C(R)$. Thus, if either $C\left(R_{1}\right)$ or $C\left(R_{2}\right)$ equals $D$ then $C(R)=D$. Therefore suppose $C\left(R_{1}\right)=\left(m_{1}, t_{1}\right)$ and $C\left(R_{2}\right)$ $=\left(m_{2}, t_{2}\right)$. By above we have $\left(\max \left(m_{1}, m_{2}\right), t_{1} \cup t_{2}\right) \leqq C(R)$.

For definiteness, let $m_{2} \geqq m_{1}$ and set $s=m_{2}-m_{1}$. By Theorem 1, there exists an $R_{1}$-module $M_{1}$, with a generating set of length $m_{1}$ and a basis of length $m_{1}+1$. Therefore $M_{1}^{\prime}=M_{1} \oplus F_{s}$, where $F_{s}$ is the free $R_{1}$-module generated by $s$ elements, has a generating set of length $m_{1}+s=m_{2}$ and basis of length $m_{1}+1+s=m_{2}+1$. There also exists an $R_{2}$-module $M_{2}$, with a generating set of length $m_{2}$ and a basis of length $m_{2}+1$. Define $R_{1} M_{2}=0$ and $R_{2} M_{1}^{\prime}=0$, then $M_{1}^{\prime} \oplus M_{2}$ is an $R$-module with a generating set of length $m_{2}$ and a basis of length $m_{2}+1$. Set $C(R)=\left(m^{\prime}, t\right)$, then what we have just shown is that $m^{\prime} \leqq \max \left(m_{1}, m_{2}\right)$. By [2, Theorem 3] $t=t_{1} \cup t_{2}$ and hence $C(R)=\left(\max \left(m_{1}, m_{2}\right), t_{1} \cup t_{2}\right)$.

\section{REFERENCES}

1. P. M. Cohn, Some remarks on the invariant basis property, Topology 5 (1966), 215-228. MR 33 \#5676.

2. W. G. Leavitt, The module type of a ring, Trans. Amer. Math. Soc. 103 (1962), 113-130. MR 24 \#A2600.

University OF Nebraska, Lincoln, Nebraska 68101 